\title{
Design Evaluation: Decomposition and State-space Analysis
}

G. Green

This paper outlines the increasing demands upon evaluation activity during the engineering design process. In particular, the need to address large numbers of innovative concept options during the conceptual design phase is stressed and a six-step methodology proposed. This methodology combines and integrates techniques of inexact reasoning with the need to combine two basic human approaches to evaluation, namely decomposition and holistic. The holistic evaluation elements comprise fuzzy estimates of probability of achieving Pareto optimal status combined with state-space analysis. An example demonstrates how these methods may converge to provide an appropriate support for human evaluation of emerging designs. It is concluded that the six-step methodology exhibits validity and time reduction in terms of providing an aid to the evaluation of a large number of merging designs and their associated design characteristics.

Keywords: evaluation, decomposition, pareto optimal, inexact reasoning, state-space.

\section{Introduction}

The ability to rapidly evaluate design ideas is an essential element in the goal to increase design productivity. Given the need for companies to produce more innovative products in an increasingly competitive market place it follows that designers have to consider an increased number of design options if the most appropriate design is to be pursued through the product development process. Only through the generation of a relatively large number of concept design options along with a rapid and reliable means of evaluating the options will designers be able to increase design productivity whilst identifying and developing new innovative products. It is recognised that a significant difficulty with evaluating design options is that they are 'information poor'. That is, important decisions often have to be taken with very limited information [1]. This provides designers with a major challenge and requires the provision of design tools and aids. These are likely to be implemented within a computer-based environment [2]. It follows that the theoretical models underpinning the design tools must be shown to be valid, reliable and robust [3].

Research activity being undertaken at the University of Glasgow is guided by a concurrent model of evaluation. It assumes two parallel strands, Holistic and Decomposition. The Holistic approach takes a complete integrated view of the design artefact and seeks to provide an evaluation of the acceptability of it. The decomposition approach, on the other hand, evaluates the design at design characteristic level and then recombines these into an overall evaluation that can then be compared with the outcome of the Holistic approach. This approach is summarised and illustrated by a six-step methodology, shown in Fig. 1. The main elements of the methodology are now briefly reviewed. These are: Decomposition of Design, Holistic Approach, Pareto Optimality.

The objectives of this paper are:

1. To outline the essential role of the evaluation activity throughout the product development process.

2. To report the current state of research activity aimed at understanding how design time can be significantly reduced via support of evaluation activity at the 'fuzzy' front-end of the design process.
3. To specifically report the status of work aimed at combining decomposition of design with space-state analysis to support human evaluation and selection of design concepts.

4. To summarise future research into design evaluation activity.

This paper therefore describes a method that models the perceived dependencies between design criteria, whilst maintaining the benefits of decomposition. This method is then linked to an approach enabling the relative estimation of the probability that a design concept will ultimately meet the requirements of the declared design criteria. This linking is shown to provide an enhanced capability to support human evaluation and selection of concept designs.

\section{Decomposition of design}

Decomposition of design is well established in practice as a means of trying to simplify the complexity of design activity [4]. Indeed recent work has even reported on the strategic decomposition approach for conceptual design [5]. Decomposition involves trying to deal with the complexity of design by both describing the required design as a set of characteristics and also by undertaking design synthesis of sub-functions rather than trying to adopt a holistic approach. The underlying assumption is that the resulting recomposed design will satisfy holistic evaluation. That is, there is an assumption of independence between the criteria. However, this is clearly not the case in most practical situations and what remains unclear is how the relationship between design characteristics should be modelled, to reflect their dependent nature, and how they may be recombined to provide a more realistic and holistic evaluation of the complete design. The models that seek to describe decomposition take as fundamental the idea of a sub-division of design space. That is, for a given design domain, the associated design characteristics $\left(D_{c h}\right)$ allow all associated specifications and models to be described in terms of the values of the characteristics. A Product Design Specification (PDS) can, in turn, be viewed as comprising a set of $D_{c h}$. The activity of evaluation then consists of the evaluator making a judgement as to whether a particular design concept will meet the target value of each $D_{c h}$ in turn. This can be illus- 


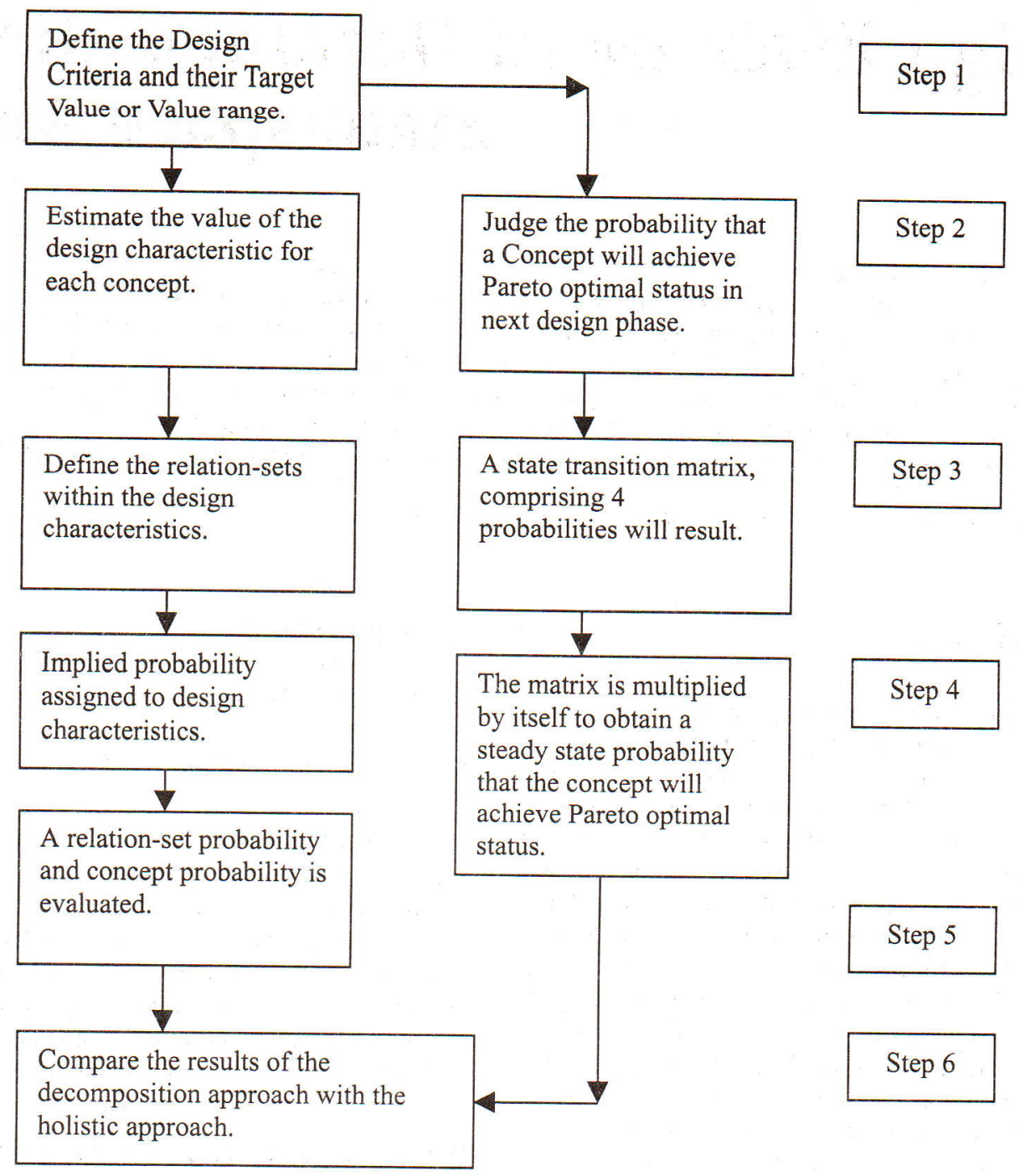

Fig. 1: Summary of evaluation methodology

trated most simply by the use of interacting uniform (rectangular) distributions, as shown in Fig. 2.

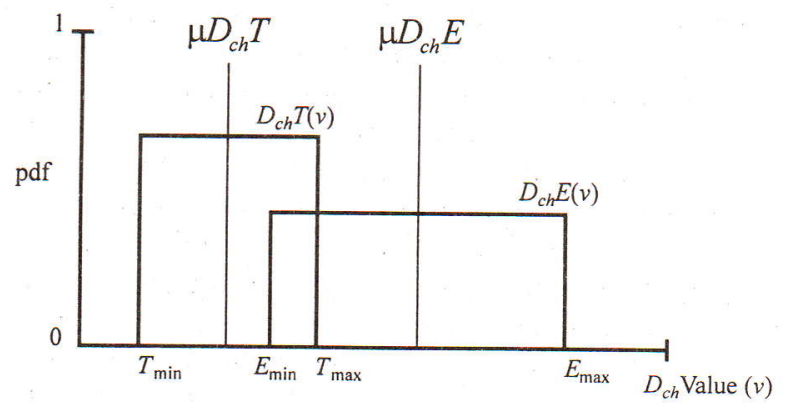

Fig. 2: Interaction of uniform distributions

The degree of interaction and hence the degree of match between the desired target value range and the estimated target range is given by the Design Margin (DM), as follows:

$$
\frac{\mu D_{c h} T-\mu D_{c h} E}{\sqrt{\left(\sigma_{T}^{2}+\sigma_{E}^{2}\right)}},
$$

where, $\mu D_{c h} T$ and $\mu D_{c h} E$ are respectively the mean values and $\sigma_{T}$ and $\sigma_{E}$ are respectively the standard deviations of the Target and Estimation distributions.

It has been shown [2] that the relationship between the design characteristics, when each is equally important, can be modelled by:

$$
\sum_{i=1}^{n}\left[\frac{\mu D_{c h} T-\mu D_{c h} E}{\sqrt{\left(\sigma_{T}^{2}+\sigma_{E}^{2}\right)}}\right] .
$$

Note: $n=$ the number of design characteristics.

In situations where it is not essential that all design characteristic targets are achieved then one possible approach is to convert the DM's to an implied probability using the standardised cumulative uniform distribution as shown in Fig. 3. In this case the distribution has been standardised over a range of 0 to 4 , thus if for example the DM has a value of 2 then the 
implied probability ( $\left.\phi_{\text {implied }}\right)$ that the target will be achieved is 0.5 .

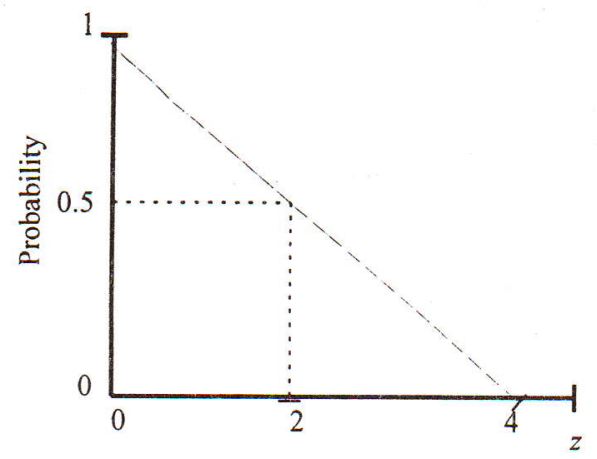

Fig. 3: Standardised cumulative uniform distribution

The values on the $\mathrm{x}$-axis represent the number of standard deviations $(z)$ of separation between a selected value $(x)$ and the mean value $\left(\mu_{x}\right)$. Four standard deviations represent almost $100 \%$ of possible values. This value $(z)$ is determined from the following equation:

$$
z=\frac{x-\mu_{x}}{\sigma_{x}}
$$

Thus, from Fig. 3, if the value of $z=0$ then there is no separation between the mean and the selected value and therefore there is $100 \%$ match and hence an implied probability value of 1 is selected. Fig. 3 is analogous to a reliability curve.

The underlying assumption here is that a 'relation-set' can be defined that links a set of design characteristics such that if at least one were to be judged on-target then there is a possibility that the design concept may develop to towards a successful conclusion. This is, analogous to a cut-set in a complex reliability network. A further assumption is that each design characteristic is independent of the others. In some situations this assumption may not be valid and therefore must be the subject of continuing research. In the meantime the following equation has been shown [3] to provide a valid method to aid identification and selection of appropriate design concepts.

$$
\sum_{i=1}^{R}\left(\prod_{i=1}^{n}\left(1-p_{\text {implied }}\right)\right)
$$

Note: $n=$ the number of design characteristics within a defined relation-set

$R=$ the number of relation-sets

Further, the relative importance of each design characteristic, usually defined within traditional evaluation methods by an individual weighting, can be seen to be inappropriate when the interaction of design characteristics takes place. A more acceptable and logical approach is to define the importance or criticality of each characteristic in terms of the desired degree of match with the design specification target levels.

\section{Holistic evaluation}

It is human nature to try to reduce complexity when at all possible. The aim is to obtain a usable answer as quickly as possible. Designers are no exception to this trait and have therefore traditionally relied upon using experience to make holistic judgements about design concepts. That is they judge the overall acceptability of a design concept rather than using methods of decomposition. This may be acceptable if the evaluator's experience is completely appropriate, but what happens to the reliability of the method when faced with new or innovative concepts? To understand this point further it is necessary to consider how a design concept progresses through the design process. We can say that a design changes state, from being an idea to a concept to an embodied design through to a detail design capable of manufacture, as it passes through the design process. So any judgement of a concept requires consideration or prediction of whether the said concept will satisfactorily progress through the process or whether it will fail to meet the demands of the design specification at some point. This is effectively the holistic judgement that is being made. State-space, in a design context, is concerned with analysing the probability of future states of a system, or design, given knowledge of the probability of the system moving between states [6], in our case during the design process. In the context of design this involves evaluating the probability that a concept will meet the inter-related requirements of a design specification and thus be judged to be in a 'working' state. As the concept progresses through phases of the design process the probability of being in a working state will vary, as information becomes richer.

A view of the likliehood of a particular concept continuing to be in a 'working' state at the end of the design process will go a long way to supporting the initial concept evaluation and selection decision. An acceptable or a working state can, in this context, be defined as Pareto Optimal. That is, $A$ concept is considered Pareto optimal if in attempting to move the value of a particular design characteristic closer to its target value or range the effect is to move another of the design criteria away from its target value or acceptable range'. In effect a Pareto Optimal state is one where you cannot make an improvement in one design characteristic without having a negative effect on another.

In making holistic judgement an evaluator is effectively having to make a subjective (fuzzy) estimate $[7,8]$ of the following:

1. Probability of maintaining a Pareto optimal state.

2. Probability of moving out with a Pareto optimal state.

3. Probability of remaining out with a Pareto optimal state.

4. Probability of moving into a Pareto optimal state.

A human evaluator makes a holistic judgement, using a 0 to 1 scale, for each concept under evaluation. For example, let us assume that the estimated probabilities are as follows: 0.9 , $0.1,0.6,0.4$. This would allow us to construct a Fuzzy Transition Probability Matrix which when multiplied by itself a sufficient number of times will reach a steady-state condition for the concept indicating its overall likliehood of being in a Pareto optimal state at the end of the process.

$$
\left[\begin{array}{ll}
0.9 & 0.1 \\
0.4 & 0.6
\end{array}\right]^{n}
$$

In this case a steady state is reached after approximately seven intervals $(n)$, this is illustrated in Fig. 4. 


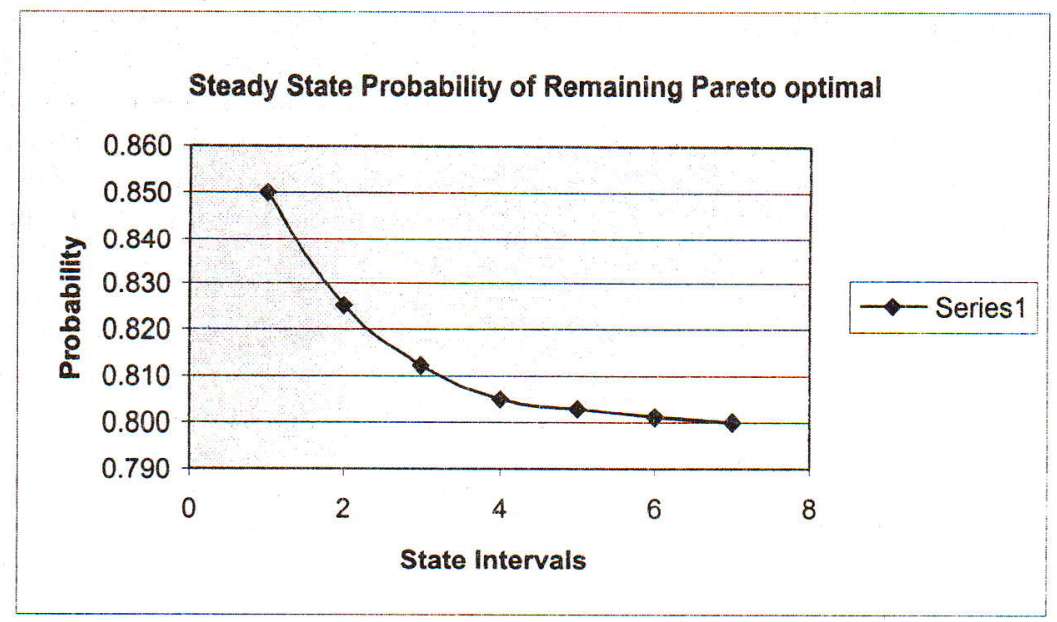

Fig. 4: Steady-state probability

Table 1: Summary of method for determination of concept scores

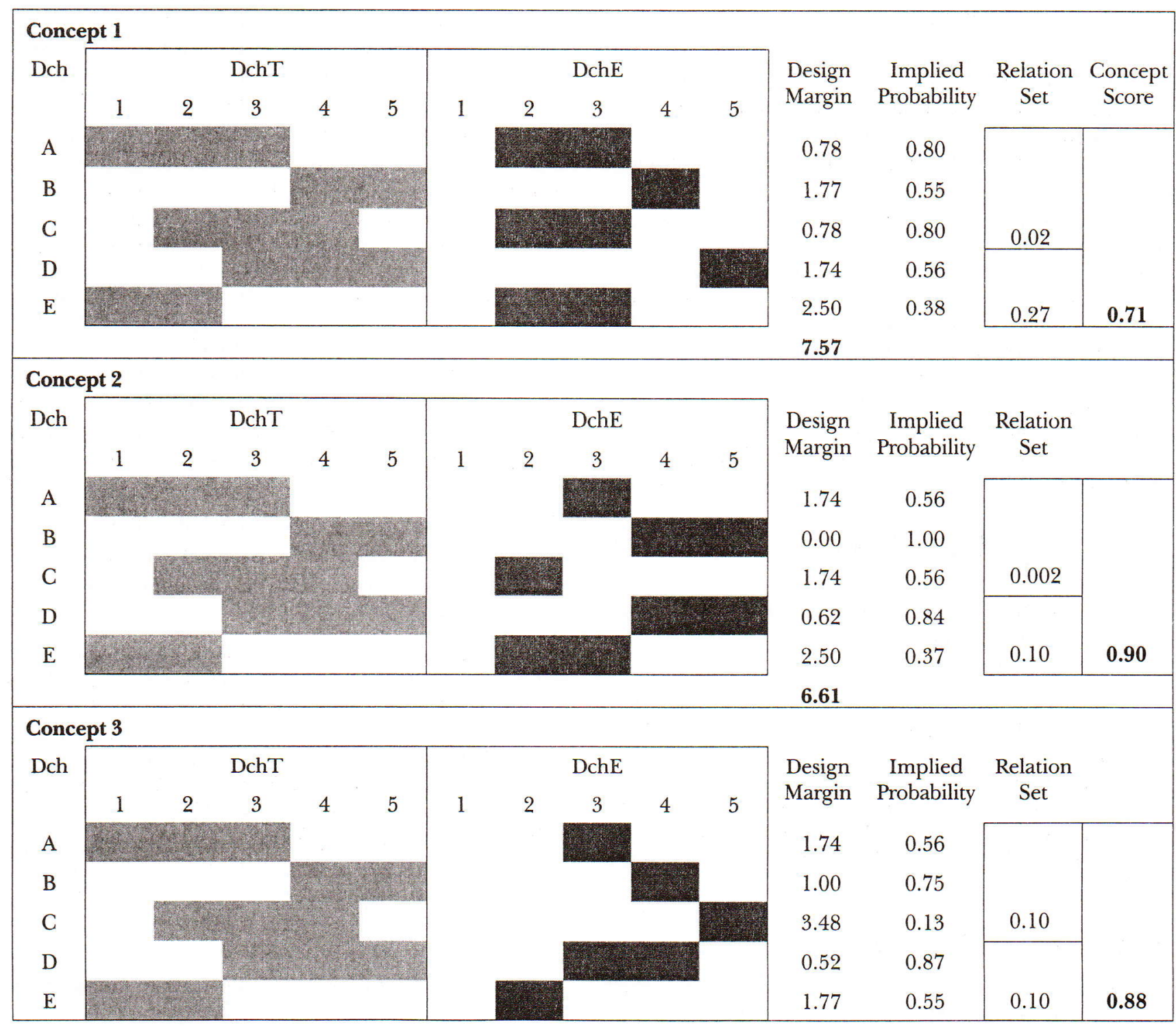


The holistic approach provides a subjective probability that the concept being evaluated will remain Pareto optimal throughout the design process. In the above case a subjective probability of 0.8 is suggested. If this figure exceeds that associated with other concepts, then this concept would be a strong contender for selection. Of course the methodology described in this paper requires that the result obtained via holistic evaluation be compared with that produced via the decomposition approach before a final conclusion is reached.

A simple example will now be used to illustrate this comparison.

\section{Example}

The following simple example is only designed to illustrate the methodology in action and does not represent a real situation. Let us therefore assume that we have produced 3 concepts as potential design solutions to a particular problem. Let us further assume that each concept is defined by 5 design characteristics (A-E).

Step 1 - In accordance with the methodology we first have to define design characteristic target $\left(D_{c h} T\right)$ values. These will obviously be the same for each concept.

Step 2 - The evaluator estimates the likely value of the Design Characteristic $\left(D_{c h} E\right)$.

Step 3 - Relation sets are determined for the Design Characteristics. In this example we assume that two relation sets exist, namely: $\{\mathrm{A}, \mathrm{B}, \mathrm{C}\},\{\mathrm{D}, \mathrm{E}\}$.

Step 4 and 5 - The Design Margin is evaluated and the implied probabilities gained from the interaction $D_{c h} T$ and $D_{c h} E$ are used in conjunction with equation 4 to obtain a measure (Concept Score) of the concept that best meets the requirements.

The results of the above are summarised in Table 1.

From the results in Table 1 we can see that the summation of Design Margins for each concept indicates that Concept 2 has the best overall match, with a score of 6.61. However, when we seek to take the relationship between design characteristics into account by using the implied probability to evaluate the relation sets we find that Concept 2 remains the best choice but that the order of the other two concepts has changed. Note that with the Design Margin approach, the smallest score is best, and with the relation set approach, the biggest score is best.

Step 6 - Requires comparison between the above and the holistic judgement using a state-space approach. Let us again assume that the evaluator's judgements about the three concepts are as summarised in Table 2.

The resultant steady-state probability of the concept remaining in Pareto optimal state is determined using the method outlined earlier in the text. We see once again that concept 2 is identified as the best of the three being evaluated. Our next step is to compare this result with those attained via the decomposition approach incorporating relation sets. To ease comparison the results are summarised in Table 3.

In the above example we see that Concept 2 has maintained dominance of the three options but that the ranking has changed from 2,1,3 with Design Margin, to 2,3,1 with the
Table 2: Summary of evaluator judgements

\begin{tabular}{|l|c|c|c|}
\hline Probability Estimates & Concept 1 & Concept 2 & Concept 3 \\
\hline $\begin{array}{l}\text { Probability } \\
\text { of maintaining a } \\
\text { Pareto optimal state }\end{array}$ & 0.9 & 0.95 & 0.85 \\
\hline $\begin{array}{l}\text { Probability of moving } \\
\text { out with } \\
\text { a Pareto optimal state }\end{array}$ & 0.1 & 0.05 & 0.2 \\
\hline $\begin{array}{l}\text { Probability } \\
\text { of remaining out with } \\
\text { a Pareto optimal state }\end{array}$ & 0.6 & 0.3 & 0.5 \\
\hline $\begin{array}{l}\text { Probability of moving } \\
\text { into a Pareto optimal } \\
\text { state }\end{array}$ & 0.4 & 0.7 & 0.5 \\
\hline $\begin{array}{l}\text { Resultant Steady-state } \\
\text { Probability }\end{array}$ & 0.80 & $\mathbf{0 . 8 6}$ & $\mathbf{0 . 7 7}$ \\
\hline
\end{tabular}

Table 3: Comparison of evaluation results

\begin{tabular}{|c|c|c|c|}
\hline & \multicolumn{2}{|c|}{ Decomposition Approach } & \multirow{2}{*}{$\begin{array}{c}\text { Holistic } \\
\text { Approach }\end{array}$} \\
\cline { 2 - 3 } & Design Margin & Relation Set & \\
\hline Concept 1 & 7.6 & 0.71 & 0.80 \\
\hline Concept 2 & 6.6 & 0.90 & 0.86 \\
\hline Concept 3 & 8.5 & 0.88 & 0.77 \\
\hline
\end{tabular}

Relation set approach and then back again to 2,1,3 with the holistic approach.

\section{Conclusions}

This paper has outlined the increasing demands upon evaluation activity during the engineering design process. In particular, the need to address large numbers of innovative concept options during the conceptual design phase has been stressed and a six-step methodology proposed. This methodology has been shown to comprise techniques of inexact reasoning with the need to combine two basic human approaches to evaluation, namely decomposition and holistic. It is clear to the author that the methods employed are only useful if they can be implemented within a computer environment, otherwise engineers and designers are likely to fall back on traditional approaches rather than the detailed approach of the six-step methodology. Only through computer implementation will the desired time reduction be achieved whilst being able to process data associated with a large number of design concepts and their associated set of design characteristics. This is work that is on-going within the Department of Mechanical Engineering at the University of Glasgow. A particular aspect of this research is the need to better model the interaction and interdependency of design characteristics and to further test the six-step methodology in active industrial environments. 


\section{References}

[1] Wu, H., Wang, F., Zhang, X., He, N.: A Model of Inexact Reasoning in Mechanical Design Evaluation. Artificial Intelligence in Engineering, Vol. 10, 1996, p. 357-362.

[2] Green, G.: Modelling Concept Design Evaluation. Artificial Intelligence for Engineering Design. Analysis and Manufacture (AIEDAM), Vol. 11, 1997, p. 211-217.

[3] Green, G.: Towards Integrated Design Evaluation: Validation of Models. Journal of Engineering Design, Vol. 11, No. 2, 2000, p. 121-132.

[4] Ho, C. H.: Some Phenomena of Problem Decomposition Strategy for Design Thinking: Differences between Novices and Experts. Design Studies, Vol. 22, No. 1, 2001, p. 27-45.

[5] Medland, A. J., Muillineux, G.: A Decomposition Strategy for Conceptual Design. Journal of Engineering Design, Vol. 11, No. 1, 2000, p. 3-16.

[6] Zhang, W. Y., Tor, S. B., Britton, G. A.: A Heuristic State-space Approach to the Functional Design of Mechanical
Systems. International Journal of Advanced Manufacturing Technology, Vol. 19, No. 4, 2002, p. 235-244.

[7] Wang, J.: A Fuzzy Outranking Method for Conceptual Design Evaluation. International Journal of Production Research, Vol. 35, No. 4, 1997, p. 995-1010.

[8] Vanegas, L. V., Labib, A. W.: Application of New Fuzzy-weighted Average (NFWA) Method to Engineering Design Evaluation. International Journal of Production Research, Vol. 39, No. 6, 2001, p.1147-1162.

Dr. Graham Green

e-mail: g.green@mech.gla.ac.uk

Department of Mechanical Engineering James Watt Building

University of Glasgow

Glasgow G12 8QQ

Scotland, United Kingdom 